\title{
Using a high spatial resolution tactile sensor for intention detection
}

\author{
Claudio Castellini \\ Robotics and Mechatronics Center \\ DLR - German Aerospace Center \\ D-82234 Weßling, Germany \\ email: claudio.castellini@dlr.de
}

\author{
Risto Kõiva \\ Neuroinformatics Group, \\ Bielefeld University \\ D-33615 Bielefeld, Germany \\ email: risto.koiva@uni-bielefeld.de
}

\begin{abstract}
Intention detection is the interpretation of biological signals with the aim of automatically, reliably and naturally understanding what a human subject desires to do. Although intention detection is not restricted to disabled people, such methods can be crucial in improving a patient's life, e.g., aiding control of a robotic wheelchair or of a self-powered prosthesis. Traditionally, intention detection is done using, e.g., gaze tracking, surface electromyography and electroencephalography. In this paper we present exciting initial results of an experiment aimed at intention detection using a high-spatial-resolution, highdynamic-range tactile sensor. The tactile image of the ventral side of the forearm of 9 able-bodied participants was recorded during a variable-force task stimulated at the fingertip. Both the forces at the fingertip and at the forearm were synchronously recorded. We show that a standard dimensionality reduction technique (Principal Component Analysis) plus a Support Vector Machine attain almost perfect detection accuracy of the direction and the intensity of the intended force. This paves the way for high spatial resolution tactile sensors to be used as a means for intention detection.
\end{abstract}

\section{INTRODUCTION}

Intention detection is the ability of a machine to reliably understand what a human wants it to do. The problem is particularly interesting for the case of people who can no longer use standard control devices such as a mouse, a keyboard, voice control, etc. In these cases, more subtle messages coming from the person's body must be interpreted; biological signals such as, e.g., surface electromyography (sEMG), gaze tracking and electroencephalography (EEG) have been used to this end. Two cases of interest are hand prosthesis, where the prosthesis replaces the hand and cannot therefore be controlled manually, and robotic wheelchairs used by patients of quadriplegia, spinal injury, muscle degenerative diseases, etc.

In such cases the user must be enabled to control aiding devices through algorithms that can interpret stable patterns from residual biological signals. For example, a wide corpus of research has concentrated on the use of surface electroencephalography to control the motion of a wheelchair, and/or to control related artifacts mounted on it, such as, e.g., a robotic arm/hand system to grasp and carry objects [1]-[3]. Of course, if no stable voluntary movement can be detected at all from the patient's body, for instance in the case of quadriplegia or locked-in syndrome, then EEG seems to be the only non-invasive means to allow intention detection.
However, for people who do have some stable, albeit, minimal residual muscular activity, then a number of techniques can be employed. This is the case, e.g., of degenerative muscular disorders (Gehrig, ALS, etc.) and various types of spinal injury; moreover, (hand) amputees are nowadays well-known to have a rich residual activity in the residual limb [4], [5].

In such cases it is desirable to find the best way for that people can express their intention and to devise adequate mathematical techniques to interpret the related signals. To this aim we have set up an experiment to check whether an advanced tactile sensor could be used for intention detection. In our experiments, we employed a custom built modular flat tactile sensor system [6] with a spatial resolution of $5 \mathrm{~mm}$ and a wide dynamic range, which was previously used in robotic studies [7], [8]. The sensor can be thought of as a window to applied forces, represented as tactile 2D images, where each taxel (tactile pixel) value holds an individual measurement of force.

The main idea is to use such a sensor to detect the changes in the force distribution at the body surface, induced by a person's attempt to express their intention. For example, trying to flex a finger should result in the thickening of the corresponding flexor muscle; this in turn should produce increased pressure on the surface of the skin, above the location of the muscle. We expect this pattern to hold until the flexion ends and the muscle tension is released. If such stable patterns of muscle activation could be effectively detected in such a way, then high spatial resolution tactile sensing would constitute a novel non-invasive human-machine interface.

In order to investigate this issue, we asked 9 able-bodied participants to exert small forces (one to four Newtons) in four directions (up, right, down, left) with their index finger, in a structured and repeatable way. During this exercise, we recorded the generated fingertip forces and tactile images of the ventral side of the forearm, gathered by our tactile sensor system. The results we present indicate that simple features extracted from the resulting images can be used to predict both the magnitude and direction of the applied force. This paves the way to use high spatial resolution tactile sensing for intention detection with severely disabled people and amputees. General feasibility of the approach, that is, its practical application, miniaturization and costs are currently 
being investigated. This approach could constitute a valid alternative to better-known and studied signals such as, e.g., gaze tracking and surface electromyography (sEMG).

The paper is structured as follows. First, we revise the related work, before describing the experimental setup and protocol in Sec. III. In Sec. IV we present the data analysis and classification results, and finally, in Sec. V we draw some conclusions and outline possible future work.

\section{COMPARISON TO RELATED WORK}

The idea of actuating a hand prosthesis via pressures exerted in the stump dates back at least to 1966 [9], when muscle bulges were used to activate a pneumatic switch and control opening / closing of the French Electric Hand. More recently, Craelius et al. have explored the issue using a hard socket fitted with 8 to 32 myo-pneumatic sensors [10] and 14 forcesensing resistors (FSRs) [11]. In both experiments, singlefinger motion discrimination was demonstrated. The same research group has lately applied the FSR-based approach (with 8 sensors) to gait control [12] and brain injury rehabilitation [13]. Gait control has also been realized this way by Lukowicz et al. [14].

The idea of detecting muscular activity via the changes induced by it on the body surface has been called in turn SMP (surface muscle pressure), FMG (force myography) and RKI (residual kinetic imaging). Of great relevance to our work is the comparison between SMP and the mainstream approach to non-invasive prosthesis control, namely, surface electromyography (sEMG), carried out in [12]. They show that, at least for the experiment considered, SMP yields a more stable and repeatable signal than sEMG with less variance and more robustness over the medium-term and across participants. The described setup is also extremely convenient, since FSRs are commercially available, affordable, small-time electronic components.

A slightly different approach, called mechanomyography (MMG) relies on the vibrations induced by muscle activation in the muscles themselves. Research in this direction (e.g., [15]-[19]) seems also very promising.

However, all these approaches lack the high spatial resolution needed to obtain a faithful representation of the activity of different muscles, which in this work we show to be important to gain a much richer and more robust insight into the subjects' intended actions.

\section{EXPERIMENT DESCRIPTION}

Participants were instructed to exert very small forces with their index finger in two axis (up/down and left/right), while a tactile-sensing board recorded the corresponding changes occurring on the ventral side of the forearm.

\section{A. Experimental Setup}

The experimental setup consisted of three main components: a two-axis force sensor, a flat tactile sensor system and the visual stimuli presented on a monitor [Fig. 1]. Along with the stimulus, live visual feedback about the exerted force was

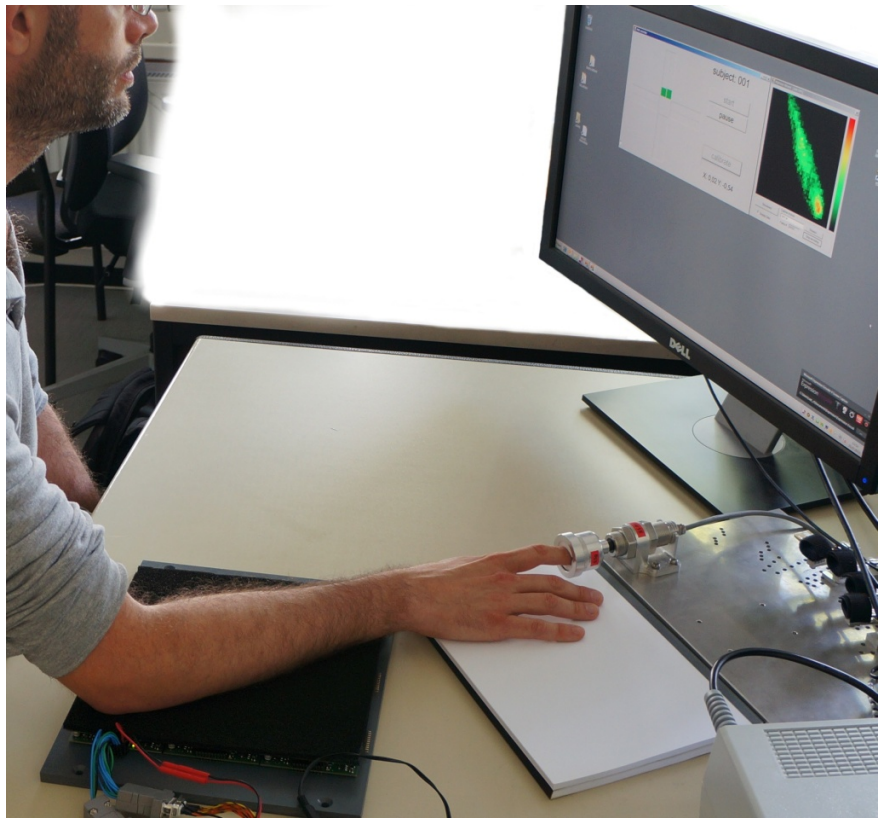

Fig. 1. The experimental setup during a trial. Flexion/extension and ab/adduction forces of the index finger are measured using a two-axis strain gauge sensor (the participant has his right index finger in the hollow of the sensor). The changes in the ventral side of the forearm are measured using a tactile sensor system (seen in black under the forearm in the figure) with $48 \times 48=2304$ tactile pixels (taxels). The stimulus, the force vector captured from the index finger by the strain gauge sensor, and the pressure pattern of the forearm are displayed on the screen.

provided to aid the participants more easily match the desired force patterns.

The index finger flexion/extension and abduction/adduction forces were gathered using the Finger-Force Linear Sensor (FFLS) [20]. The sensing is based on a highly accurate industrial radial dual axis strain gauge sensor with a $\pm 100 \mathrm{~N}$ range. The sensor outputs are passed to two strain-gauge amplifiers, connected to a data acquisition card in the computer. The index finger was attached to the dual-axis sensor using different sized gypsum casts, allowing a slack-free fit between the finger and the sensor.

In order to capture muscle bulges on the ventral side of the arm we used a $3 \times 3$ array of modular tactile sensors [6]. Each of the modules has $16 \times 16$ tactile pixels (taxels), resulting in a total of $48 \times 48=2304$ taxels covering an area of $240 \times 240 \mathrm{~mm}$ [Fig. 2]. The tactile sensor is based on a resistive sensing working principle with a characteristic hyperbolic output, explained in detail in [21]. This allows a fine-grained insight into subtle hand movements, while still being able to output discriminating values for high finger forces (with a main measurement range between 1 and $30 \mathrm{kPa}$ ). The internal analog-digital-converters have a resolution of 12bits and output data at approximately $264 \mathrm{~Hz}$ each. The tactile sensor is connected to a PC via USB 2.0 employing the USBVideo-Class protocol used by USB-Webcams, and delivering a video stream of tactile data to the host computer, where each pixel of a frame corresponds to a pressure value of a taxel. 


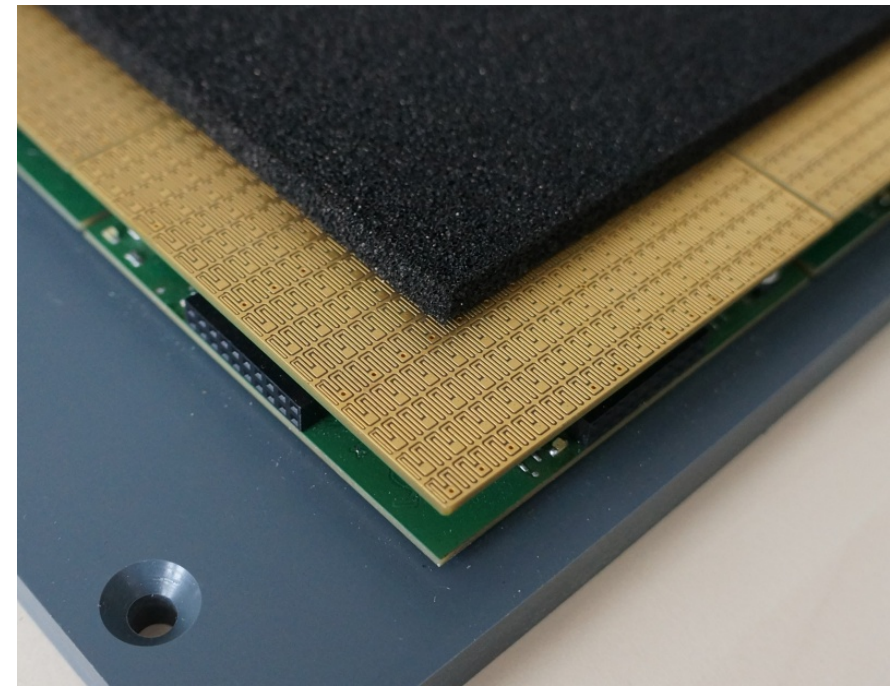

Fig. 2. Detail of the tactile sensor system [6] used in the experimental setup. The sensor has $5 \mathrm{~mm}$ spatial resolution; nine modules with $80 \times 80 \mathrm{~mm}$ and $16 \times 16$ tactile cells each are used in a $3 \times 3$ panel configuration.

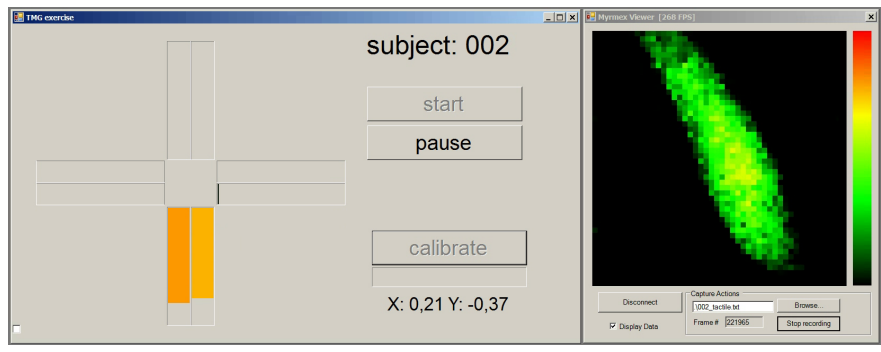

Fig. 3. Screenshot of the visual display shown to the participant during the trial. The stimulus direction and amplitude are displayed as a cross in the left window. Next to the stimulus bars, the live-captured values of the two-axis strain gauge sensor are shown as a feedback to the user. On the right, the live pressure pattern of the arm, as captured by the tactile sensor system, is visualized.

The stimulus was presented to the participants on a large computer monitor. It consisted of a set of colored bars displaying the requested force pattern of the index finger. The layout and colors were chosen to be as intuitive as possible; the length and the color-coding of the bars denoted the intensity of the force, and the orientation denoted the direction (e.g., a bar moving up would induce the participant to apply a force upwards, that is to extend the index finger; a bar moving right would induce index adduction, etc.).

Alongside the stimulus bars, feedback bars displaying the measured force magnitudes and directions were shown in realtime, so that the task was reduced to that of matching the stimulus bars [Fig. 3 shows a screenshot of the stimulus].

\section{B. Experimental Protocol}

Nine able-bodied, healthy subjects (all male, right-handed, aged $29.9 \pm 5.1 \mathrm{yrs})$ participated in the experiment, which lasted about 15 minutes. Each subject sat comfortably on an office chair in front of the monitor; the chair was adjusted for each participant to obtain maximum comfort. The participant's right

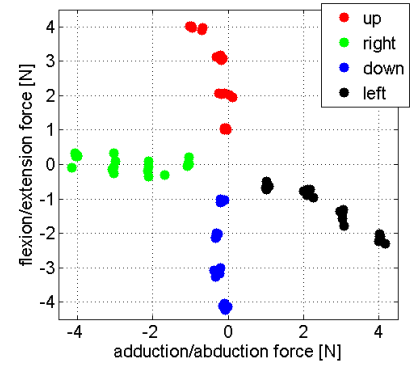

(a) Force Directions.

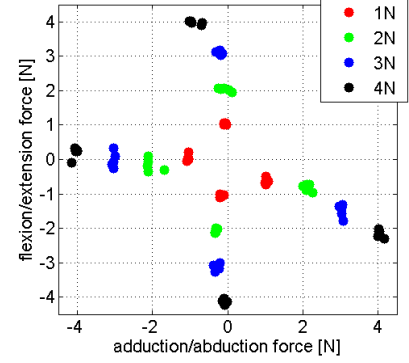

(b) Force Magnitudes.
Fig. 4. Typical captured index finger force data plotted on a 2D-graph. (a) displays the clustering of captured data in force directions, (b) displays the data from the same experiment, clustered according to force magnitudes. Notice the skewness of the adduction forces [Fig. 4a, black samples]. In this case the participant could not avoid flexing the index while adducting.

forearm was placed on the tactile sensor while their right index finger was inserted into the dual-axis force sensor. No physical measure was taken to prevent participants from moving their forearm during the experiment (although, they were instructed to try to not move it explicitly).

The stimulus consisted of four levels of desired forces, 1 to $4 \mathrm{~N}$ (in $1 \mathrm{~N}$ increments), applied either up (index finger extension), right (adduction), down (flexion) or left (abduction). During the experiment, each force magnitude and direction combination was repeated 7 times (in a non-random manner), in order to generate a learning effect.

\section{EXPERIMENTAL RESULTS}

\section{A. Qualitative Analysis}

For the qualitative analysis and the classification of forces, the first two repetitions of each induced pattern were discarded from the dataset. All in all, this resulted in $5 \times 4 \times 4 \times 9=$ 720 patterns, each one labeled according to the direction, magnitude and repetition number. Each force pattern stimulus lasted 5 seconds, with 3 seconds of rest in-between. Data gathered from the tactile and force sensors, plus data from the stimulus were synchronized using timestamps provided by the CPU. For each pattern, we averaged all values during the middle third of the 5 second pattern duration, in order to ensure only stable data was considered. This resulted in one sample per pattern, that is, 80 samples per participant.

We first validated and verified the acquired index finger dual-axis force sensor data via visual inspection by plotting the force data on a 2D-graph, which faithfully represent the gathered patterns. Fig. 4 displays a typical example of such a graph.

As one can see, the force vectors are perfectly clustered according to either the required force direction or the magnitude. This is true for all participants, notwithstanding slight distortions of the clusters due to each participant's anatomy. In Fig. 4a, for example, this participant could not avoid flexing the index finger during adduction - this is reflected in the inclination of the black cluster in the graph. 


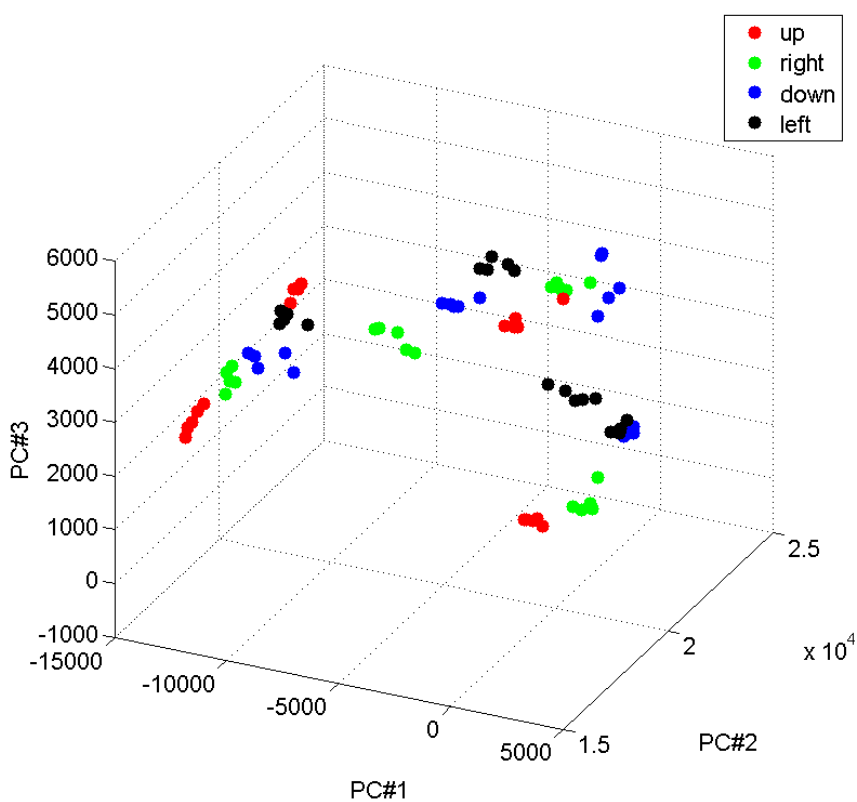

(a) Force Directions.

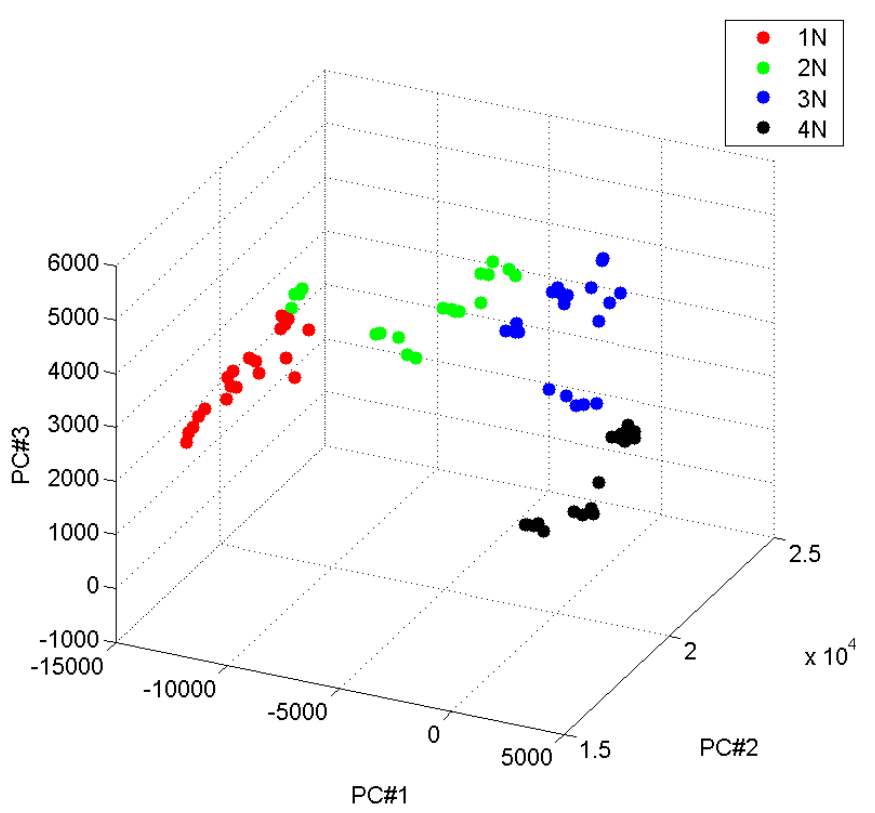

(b) Force Magnitudes.

Fig. 5. 3D-PCA-projected tactile data for a typical subject.

Much less obvious is the fact that a similar phenomenon might happen in tactile space; actually, this turns out to be the case. The images gathered from the sensor consist of 2304 taxels $(48 \times 48)$. We applied PCA on the dataset obtained (80 samples for 9 participants, which equates to 720 samples) and discovered that 5 principal components accounted for $89.9 \% \pm 3.65 \%$ of the signal variance (mean plus/minus one standard deviation over all participants). This is not surprising, as the participants were uniformly asked to only perform 4 actions. In order to visually observe if the samples are somewhat clustered, the resulting data can be succinctly plotted on a 3D graph if we retain only three components [Fig. 5].

As can be seen from Fig. 5b, the samples appear almost perfectly clustered according to the force magnitudes. As opposed to that, clustering according to force direction [Fig. 5a] appears less clear, but one should note that, within each magnitude cluster, the direction sub-clusters are clearly separable. This means that even a simple classification system should be able to tell the force magnitudes and directions apart, just by considering the tactile sensor array samples.

\section{B. Classification of force magnitude and direction}

We have employed a Radial-Basis-Function (RBF) SVM [22], [23] to classify the tactile samples according to force magnitude and direction. Leave-One-Out error evaluation was used since the number of samples is relatively small. Table I shows the results.

The table clearly shows that classification is in many cases perfect or near perfect, and that we did not observe a classification error higher than $6.25 \%$. (Perfect classification in this case is justified by the relatively small number of samples
TABLE I

CLASSIFICATION ERRORS FOR FORCE MAGNITUDES AND DIRECTIONS, AND RELATED STATISTICS.

\begin{tabular}{|r|rr|}
\hline Participant\# & Direction error & Magnitude error \\
\hline 1 & $1.25 \%$ & $0 \%$ \\
\hline 2 & $0 \%$ & $0 \%$ \\
\hline 3 & $6.25 \%$ & $2.5 \%$ \\
\hline 4 & $0 \%$ & $1.25 \%$ \\
\hline 5 & $1.25 \%$ & $2.5 \%$ \\
\hline 6 & $1.25 \%$ & $0 \%$ \\
\hline 7 & $0 \%$ & $0 \%$ \\
\hline 8 & $1.25 \%$ & $0 \%$ \\
\hline 9 & $0 \%$ & $0 \%$ \\
\hline mean \pm std. & $1.25 \% \pm 1.98 \%$ & $0.69 \% \pm 1.10 \%$ \\
\hline
\end{tabular}

available.)

\section{Prediction of forces}

Next we wanted to test whether the tactile images could be used to predict the exact amount of exerted forces and the direction depending on the magnitude and direction of the stimulus. To this end, four regions of interest (ROIs) were identified on the tactile image,

$$
\mathrm{ROI}_{i}=\left\{(x, y):\left|x-x_{i}\right| \leq r,\left|y-y_{i}\right| \leq r\right\}
$$

where $i=1, \ldots, 4$ and $r=7$ taxels. (Notice that the point locations and the regions are the same across all images and participants.) Then for each ROI a linear spatial approximation (plane) of the tactile force values was evaluated:

$$
\left.g(x, y)\right|_{(x, y) \in \mathrm{ROI}_{i}} \approx \alpha_{i}\left(x_{i}-x\right)+\beta_{i}\left(y_{i}-y\right)+\gamma_{i}
$$




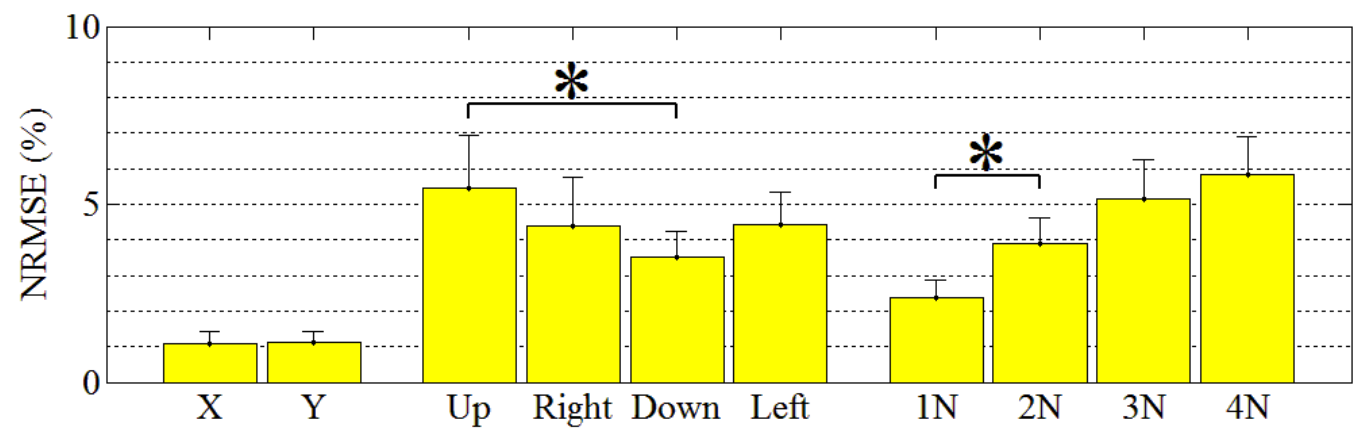

Fig. 6. The NRMSE obtained while predicting finger forces using the spatial linear approximations of the tactile images. (left) Global values for sensor directions X (index ab-/adduction) and Y (flexion/extension); (middle) Values aggregated over force directions; (right) Values aggregated over force magnitudes. The asterisks denote two pairs of statistically significant (Student's t-test, $p<0.01$ ) error rates.
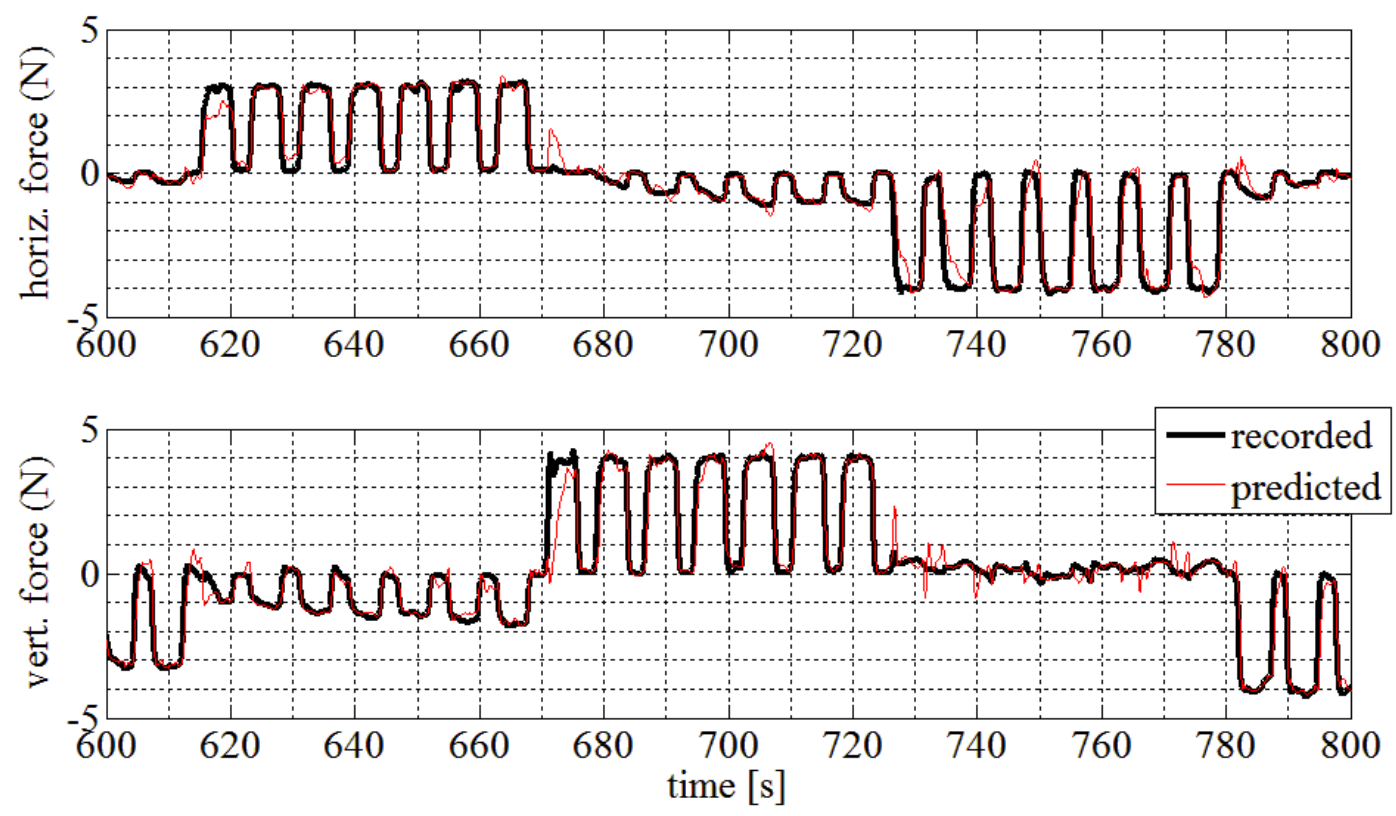

Fig. 7. Exemplar recorded and predicted forces for participant \#9. (upper panel) Along the finger ab-/adduction direction (horiz. force); (lower panel) Along the finger flexion/extension direction (vert. force). The resulting ab-/adduction and flexion/extension NRMSE are $1.14 \%$ and $1.31 \%$.

where $g(x, y)$ denotes the force value at taxel coordinates $(x, y)$. This way twelve features, i.e., $\alpha_{i}, \beta_{i}$ and $\gamma_{i}$ for $i=$ $1, \ldots, 4$, were extracted from each image (this set of features resembles the one employed in [24], [25] where ultrasound images of the forearm were considered). The resulting set of features, along with the force data from the strain gauge sensor, were low-pass filtered $\left(1^{\text {st }}\right.$ order Butterworth filter, cutoff frequency of $1 \mathrm{~Hz}$ ) and subsampled at $25 \mathrm{~Hz}$, resulting in about 23,000 tactile/force pairs.

In order to check whether the force could be predicted from the tactile data, we trained two RBF SVMs for each participant, one for the force on the $\mathrm{X}$ axis of the sensor (index ab-/adduction) and one for the $\mathrm{Y}$ axis (flexion/extension), this time in $\epsilon$-regression mode (see, e.g., [26]). As the number of samples was extremely large, we picked one sample in 50 for the training set, which was used to evaluate, via 5-fold cross-validation and grid-search, the optimal hyperparameters. A model trained with these parameters on the whole training set was then used to predict the force values on the remaining sample/target pairs. Notice that this accounts for only 460 samples out of $23,000,2 \%$ of the whole dataset.

Fig. 6 shows the results for pooled abduction/adduction (X) and pooled flexion/extension (Y) force vector prediction errors, aggregated according to the force direction (Up,Right,Down,Left) prediction errors and force magnitude errors $(1 \mathrm{~N}$ to $4 \mathrm{~N})$. Error bars denote the average plus/minus one standard deviation, evaluated over all participants. As a measure of error we chose the normalized root-mean-square error (NRMSE) over the range of measured index finger forces (about $8.5 \mathrm{~N}$ ). The global NRMSEs are $1.09 \% \pm 0.35 \%$, along the index finger ab-/adduction direction and $1.12 \% \pm 0.31 \%$ along the flexion/extension direction (these values are partic- 

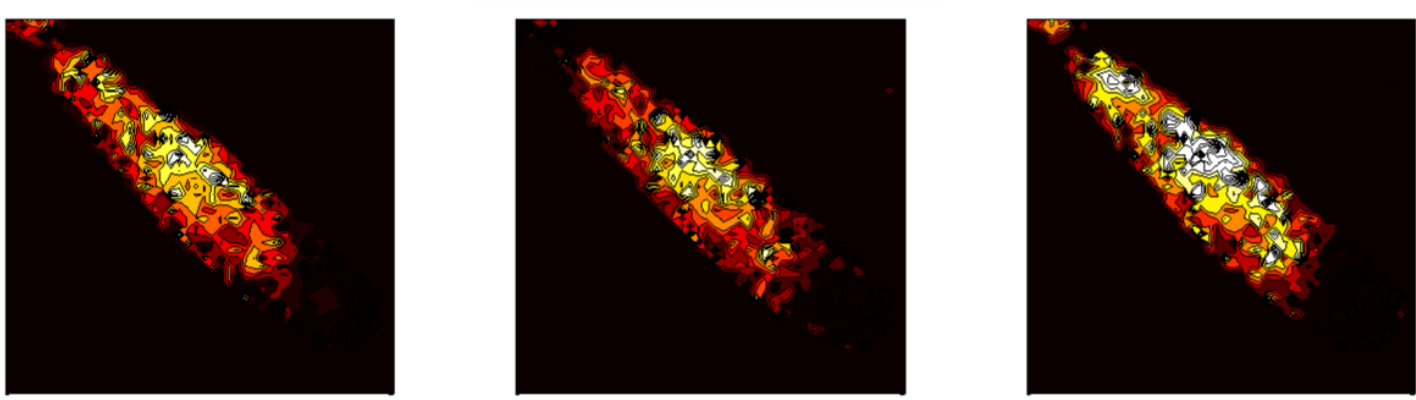

\section{$4 \mathrm{~N}$ left, 3 repetitions}
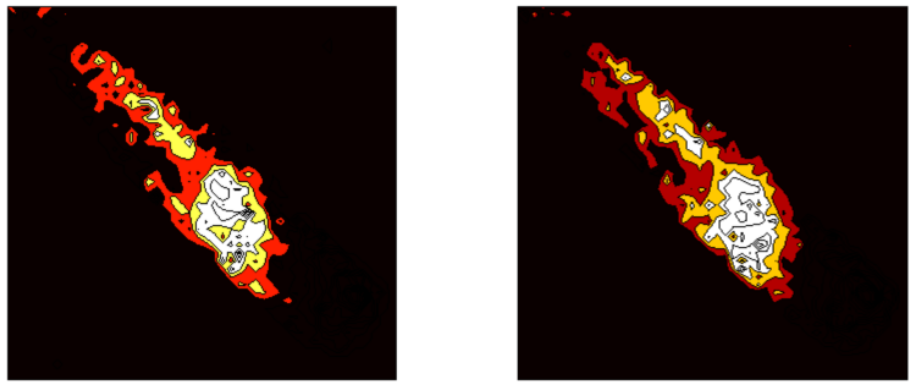

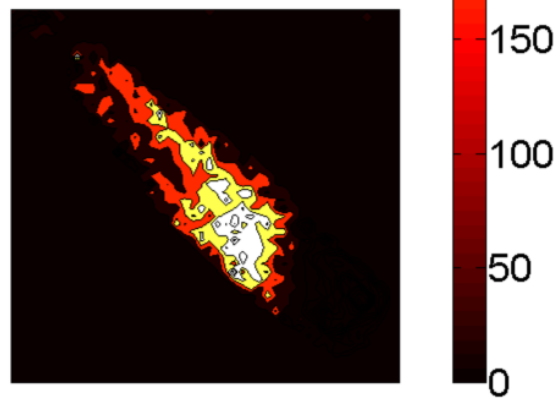

Fig. 8. Differential tactile images of a typical participant's forearm while exerting a $4 \mathrm{~N}$ force with the index finger towards up (top row) and towards left (bottom row). Common muscle activations are clearly visible. (The force scale displayed is a unitless 12-Bit value, as transmitted by the tactile sensor system.)

ularly low since they include all the resting phases). As far as force direction is concerned, whereas there seems to be no difference between abduction and adduction ("Left" and "Right" columns in the Figure), there is a statistically significant difference between extension (column "Up", NRMSE $5.46 \% \pm 1.48 \%$ ) and flexion (column "Down", NRMSE $3.51 \% \pm 0.74 \%$ ). This might be due to the fact that extending a finger is intrinsically harder to do than flexing it, which leads to a lower signal-to-noise ratio. Finally, as far as force magnitudes are concerned, notice that the NRMSE increases as the forces increase. Taking into account that the range of forces was about $8.5 \mathrm{~N}$ in both measured index finger movement axis, this evaluates to an absolute error of about one fifth of a Newton when the applied forces were $1 \mathrm{~N}$ to about half a Newton for the $4 \mathrm{~N}$ forces. Fig. 7 shows exemplar true and predicted forces.

\section{Tactile Forearm Imaging}

Finally, although no detailed image analysis was thus far performed, we show that the tactile images indeed possess anatomically related features, and that these features are common across force patterns.

Fig. 8 shows six exemplary tactile images of a participant engaged in the exertion of $4 \mathrm{~N}$ forces. The images are obtained during three repetitions of the force-up and force-left patterns, while the resting state was extracted from the force images. In the top row a high-pressure zone is clearly seen in the middle region of the forearm, while the elbow (situated in the bottom-right corner of the images) does not produce much of an effect; this is consistent with a strong extension of the index finger, during which a reaction force towards the bottom appears. In contrast to this, consider the bottom row, in which two high-pressure regions appear near the elbow and over the distal section of the forearm (upper-right corner of the arm footprint in the images), again consistent with the exertion of a finger force directed to the left. Notice that these activation patterns are visually consistent across the repetitions.

\section{CONCLUSIONS AND FUTURE WORK}

Given the results presented in this paper, we claim that a high-resolution tactile sensor could be used as an effective human-machine interface, for detecting the intention of an amputee or of a severely disabled person in a wheelchair. Our results indicate that this approach can detect small forces (one to four Newtons) to a high degree of accuracy, up to perfect classification in a significant number of cases, and up to one quarter of a Newton during force regression. These results are consistent across all nine participants engaged in the experiment. A qualitative examination of the tactile images also shows that the deformation in the arm is clearly and consistently detectable from the captured and processed visual tactile pattern. The fact that the approach can detect such small forces means that a related action could be triggered by minimal muscular residual activity. Notice that, even in the 
absence of clear manifestations of the forces exerted (e.g., no motion at the fingertips due to spinal injury), internal musculoskeletal movements and forces can still be detected. In such a case, in which no force/torque sensor can be used (but our approach could still be trained using the stimulus itself) this idea could be therefore successfully employed, e.g., in robotic wheelchair control.

As far as future work is concerned: the system shows potential to be miniaturized and to be made portable; moreover, it can be applied anywhere on the participant's body, where minimal stable residual musculoskeletal activity can be detected. Its high spatial resolution might enable very fine control over single fingers of dexterous hand prosthesis. Although with the setup we have used here it is impossible to discriminate muscle bulging from forearm spurious motion, we believe that in a different arrangement such a sensor could detect an amputee's intention to an unprecedented degree of detail; one idea is that of arranging patches of the above described tactile sensor around an amputee's stump, as in a bracelet.

\section{ACKNOWLEDGMENTS}

The authors would like to thank Carsten Schürmann of Bielefeld University for developing the modular tactile sensor system. We would like to thank Patrick van der Smagt of the Technische Universität München and Michael Suppa of the DLR for their continual financial, moral and logistic support. This work was partially supported by the DFG Center of Excellence EXC 277: Cognitive Interaction Technology (CITEC).

\section{REFERENCES}

[1] Y. Matsumoto, T. Ino, and T. Ogasawara, "Development of intelligent wheelchair system with face and gaze based interface," in IEEE/RSJ International Conference on Intelligent Robots and Systems (ROMAN), Bordeaux and Paris, France, 2001, pp. 262-267.

[2] R. Leeb, H. Sagha, R. Chavarriaga, and J. d. R. Millán, "A hybrid braincomputer interface based on the fusion of electroencephalographic and electromyographic activities," J. Neural Eng., vol. 8, 2011.

[3] L. A. Rivera and G. N. DeSouza, "A Power Wheelchair Controlled using Hand Gestures, a Single sEMG Sensor, and Guided Under-determined Source Signal Separation," in IEEE RAS \& EMBS International Conference on Biomedical Robotics and Biomechatronics (BioRob), Rome, Italy, June 2012.

[4] C. Castellini, E. Gruppioni, A. Davalli, and G. Sandini, "Fine detection of grasp force and posture by amputees via surface electromyography," Journal of Physiology (Paris), vol. 103, no. 3-5, pp. 255-262, 2009.

[5] F. V. Tenore, A. Ramos, A. Fahmy, S. Acharya, R. Etienne-Cummings, and N. V. Thakor, "Decoding of individuated finger movements using surface electromyography," IEEE Trans. Biomed. Eng., vol. 56, no. 5, pp. $1427-1434,2009$.

[6] C. Schürmann, R. Kõiva, and R. Haschke, "A modular high-speed tactile sensor for human manipulation research," in IEEE World Haptics Conference (WHC), Istanbul, Turkey, June 2011.

[7] M. Schöpfer, C. Schürmann, M. Pardowitz, and H. J. Ritter, "Using a piezo-resistive tactile sensor for detection of incipient slippage," in International Symposium on Robotics, VDE. Munich, Germany: VDE, June 2010.
[8] C. Schürmann, R. Kõiva, R. Haschke, and H. J. Ritter, "Analysis of human grasping under task anticipation using a multi sensory tactile book," in IEEE-RAS International Conference on Humanoid Robots (Humanoids 2012), Osaka, Japan, Nov. 2012.

[9] L. F. Lucaccini, P. K. Kaiser, and J. Lyman, "The French electric hand: Some observations and conclusions," Bulletin of Prosthetics Research, vol. 10, no. 6, pp. 31-51, 1966.

[10] D. J. Curcie, J. A. Flint, and W. Craelius, "Biomimetic finger control by filtering of distributed forelimb pressures," IEEE Transactions on Neural Systems and Rehabilitation Engineering, vol. 9, no. 1, pp. 69-75, 2001.

[11] M. Wininger, N.-H. Kim, and W. Craelius, "Pressure signature of forearm as predictor of grip force," Journal of Rehabilitation Research and Development, vol. 45, no. 6, pp. 883-892, 2008.

[12] D. A. Yungher, M. T. Wininger, J. B. Barr, W. Craelius, and A. J. Threlkeld, "Surface muscle pressure as a measure of active and passive behavior of muscles during gait," Medical Engineering \& Physics, vol. 33, pp. 464-471, 2011.

[13] D. Yungher and W. Craelius, "Improving fine motor function after brain injury using gesture recognition biofeedback," Disability and Rehabilitation: Assistive Technology, pp. 1-5, 2012.

[14] P. Lukowicz, F. Hanser, F. Szubski, and W. Schobersberger, "Detecting and interpreting muscle activity with wearable force sensors," in Pervasive Computing 4th International Conference (PERVASIVE), Dublin, Ireland, 2006, pp. 101-106.

[15] C. Orizio, Surface mechanomyogram. Wiley-IEEE Press, 2004, pp. 305-322.

[16] Y. Nolan and A. dePaor, "The mechanomyogram as a channel of communication and control for the disabled," in Engineering in Medicine and Biology Society, 2004. IEMBS '04. 26th Annual International Conference of the IEEE, vol. 2, Sept. 2004, pp. 4928-4931.

[17] Z. F. Yang, D. Kumar, and S. Arjunan, "Mechanomyogram for identifying muscle activity and fatigue," in Engineering in Medicine and Biology Society, 2009. EMBC 2009. Annual International Conference of the IEEE, Sept. 2009, pp. 408-411.

[18] T. W. Beck, Application of Mechanomyography for Examining Muscle Function. Transworld Research Network, 2010.

[19] J. M. DeFreitas, T. W. Beck, and M. S. Stock, "Effects of strength training on mechanomyographic amplitude," Physiological Measurement, vol. 33, no. 8, p. 1353, 2012. [Online]. Available: http://stacks.iop.org/0967-3334/33/i=8/a=1353

[20] R. Kõiva, B. Hilsenbeck, and C. Castellini, "FFLS: An accurate linear device for measuring synergistic finger contractions," in IEEE Engineering in Medicine \& Biology Society $(E M B C)$, San Diego, USA, Aug. 2012.

[21] K. Weiss and H. Wörn, "The working principle of resistive tactile sensor cells," in IEEE International Conference Mechatronics and Automation (ICMA 2005), vol. 1, Niagara Falls, Canada, July 2005, pp. 471-476.

[22] B. E. Boser, I. M. Guyon, and V. N. Vapnik, "A training algorithm for optimal margin classifiers," in 5th Annual ACM Workshop on Computational Learning Theory (COLT), D. Haussler, Ed. Pittsburgh, PA, USA: ACM press, 1992, pp. 144-152.

[23] N. Cristianini and J. Shawe-Taylor, An Introduction to Support Vector Machines (and Other Kernel-Based Learning Methods). CUP, 2000.

[24] C. Castellini and G. Passig, "Ultrasound image features of the wrist are linearly related to finger positions," in International Conference on Intelligent Robots and Systems (IROS), San Francisco, CA, USA, 2011, pp. 2108-2114.

[25] C. Castellini, G. Passig, and E. Zarka, "Using ultrasound images of the forearm to predict finger positions," IEEE Transactions on Neural Systems and Rehabilitation Engineering, vol. 20, no. 6, pp. 788-797, 2012.

[26] A. J. Smola and B. Schölkopf, "A tutorial on support vector regression," Statistics and Computing, vol. 14, pp. 199-222, 2004. 